\title{
Next day telephone follow up of the elderly: a needs assessment and critical incident monitoring tool for the accident and emergency department
}

\author{
H D M Poncia, J Ryan, M Carver
}

\begin{abstract}
Background-Patients over the age of 75 years comprise an increasing proportion of accident and emergency ( $A \& E$ ) department attendances. Within this group there is a high incidence of comorbidity, which mandates effective discharge coordination from the $A \& E$ department.
\end{abstract}

Objectives-The aims of this study were to assess the needs of these patients the day after discharge, target patients for appropriate interventions and identify critical incidents.

Setting-The study was undertaken in a district general hospital A\&E department that has 62000 new patient attendances per year.

Inclusion criteria-Patients aged 75 years or over who were discharged from the A\&E department.

Exclusion criteria-Nursing home patients. Patients without a telephone.

Study design-Pre-discharge information was collected from the medical notes. A community liaison nurse (CLN) then contacted patients by telephone. A semistructured questionnaire was used to assess patients. Patients were risk stratified and appropriate interventions made. Interventions initiated by the CLN were scored from 1 to 6 based on the level of input required.

Results-551 patients or their carers were contacted by telephone. Existing home support was felt to be insufficient in 44 $(8 \%)$ cases and in need of immediate intervention in a further $45(8 \%)$ cases. Sixty five (11\%) Category 1 patients required no intervention, 223 (42\%) Category 2 patients required advice only, 107 $(19 \%)$ Category 3 patients were referred to their GP, 127 (23\%) Category 4 patients required a domicillary visit by a GP or a nurse, 26 (5\%) Category 5 patients were at risk requiring urgent home assessment and three Category 6 patients had to re-attend $A \& E$. Advice was given by the CLN on a broad range of issues and a wide range of health care services was accessed. Five hundred and fifty nine referrals were made by the CLN after telephone assessment.

Conclusions-Telephone follow up of patients over 75 attending our $A \& E$ department identified a number of areas where care could be improved before and after discharge. This low cost, high quality intervention has the potential for decreasing inappropriate return visits to the department by a vulnerable group of patients as well as improving overall quality of care.

(F Accid Emerg Med 2000;17:337-340)

Keywords: elderly; telephone follow up

One in six people in the United Kingdom is aged over 65 years. This is expected to increase to one in five by the year 2021. ${ }^{1}$ Patients aged over 75 constitute $10-14 \%$ of accident and emergency (A\&E) attendances and $36 \%$ of all hospital admissions. There is a dearth of research and education in geriatric emergency medicine. ${ }^{2}$ Elderly patients require more time and resources than other adult patients. ${ }^{3}$ The social and personal concerns of the elderly are frequently not met and emergency physicians often find it harder to treat elderly patients than younger patients with the same complaints. ${ }^{2}$ Many factors contribute to the increasing numbers of elderly patients attending $\mathrm{A} \& \mathrm{E}$ departments including demographic shifts, social changes and shorter hospital stays. ${ }^{45}$ Elderly patients discharged from A\&E departments are a vulnerable group with health and social dependency after discharge occurring in up to $50 \% .^{6-8}$ Furthermore up to $6 \%$ of elderly patients discharged from A\&E departments are re-admitted within 14 days after a functional deterioration.

The national review of $A \& E$ services by the Audit Commission in 1996 found that $30 \%$ of A\&E departments were discharging frail, elderly patients with no follow up care and recommended that A\&E departments should screen and follow up this group of patients. ${ }^{9}$ The Health of the Nation document also set a target for a reduction in accidents of at least 33\% among people aged over 65 by the year $2005 .^{10}$ The early detection of a deteriorating situation may avoid hospital admission and delay the move into residential accommodation. ${ }^{11}$ Consequently, the identification of at risk patients, and early, targeted, multidisciplinary interventions to minimise risk without compromising functional independence should be a high priority in the health care of the elderly. ${ }^{12}$

For these reasons we conducted a study of next day telephone follow up of elderly patients. The objectives of this study were to assess the degree to which next day telephone follow up can be used as a needs assessment tool within an A\&E department. We also wished to identify those patients who we 
Table 1 Criteria for entry into Categories 1-6

\begin{tabular}{ll}
\hline Category & Entry criteria \\
\hline 1 & No issues identified. \\
& No issues identified requiring primary health care (PHC) intervention. \\
& CLN advice only required. \\
& Issues identified requiring non-urgent PHC assessment; \\
- Discharge policy not compliant with A\&E guidelines or \\
- No support identified or \\
- Unable to contact patient/carer or \\
- Unclear documentation regarding: other injuries sustained, circumstances of fall, level of \\
personal support. \\
Additional medical or social issues identified requiring more urgent investigation by PHC; \\
- More than one Category 3 entry criteria or \\
- Patient re-attended with same complaint during previous 6 months. \\
- Patient sent home unaccompanied after 9 pm. \\
- Complex liaison work with other agencies. \\
Immediate medical or social problems for patient or carer identified that require urgent action by \\
PHC; \\
- More than two Category 3 entry criteria. \\
- Condition deteriorated between discharge and telephone contact. \\
Presenting issues indicate urgent consultation necessary with A\&E consultant for possible review.
\end{tabular}

considered to be most at risk of being involved in a critical incident and to target multidisciplinary interventions early to those who might benefit the most.

\section{Methods}

The study period was from November 1997 to April 1998. The notes of all patients aged 75 or over who were discharged from the A\&E department the previous day were reviewed the day after attendance. Information collected included mode of arrival, referral source, home telephone number, type of accommodation, existing support at home and advice given by staff. The patient was then contacted by telephone by a community liaison nurse (CLN). A semi-structured questionnaire was used to assess the current level of disability, if any deterioration had occurred since discharge, the type of care received at home, compliance with medication and patient comprehension. ${ }^{13}$ Assessments of patient comprehension were subjective, however all telephone follow up calls and assessments were made by a single CLN. Patients were telephoned the next day except those who had attended over the weekend, who were contacted on the following Monday. To assess the impact of the telephone interventions we developed a scoring system where patients were risk stratified according to perceived levels of risk based on physical, emotional and social needs and according to a number of key risk criteria (table 1). Category 1 patients required no intervention. Category 2 patients required advice only. Category 3 patients were felt to be at potential risk and in need of general practitioner (GP) follow up. Category 4 patients were felt to be at potential risk but in need of a more urgent domicillary visit by either a GP, nurse or occupational therapist

Table 2 Intervention according to risk category

\begin{tabular}{lcl}
\hline Category & $\begin{array}{l}\text { Number } / \% \\
(n=551)\end{array}$ & Intervention \\
\hline 1 & $65(12)$ & None \\
2 & $223(40.5)$ & Advice only by CLN \\
3 & $107(19)$ & Patient advised to see GP \\
4 & $127(23)$ & GP or nurse to visit \\
5 & $26(5)$ & At risk-urgent home assessment \\
6 & $3(0.5)$ & To re-attend A\&E department \\
\hline
\end{tabular}

within 48 hours. Patients were entered into Category 5 if they were considered to be at risk and in need of immediate intervention. After telephone assessment appropriate interventions were made (table 2).

\section{Results}

Five hundred and sixty five patients over 75 were discharged from the department during the study period. A total of 551 patients were followed up by telephone.

\section{INITIAL A\&E ATTENDANCE}

GPs had referred $274(50 \%)$ patients to the A\&E department. Some 334 (61\%) had arrived by ambulance. Altogether 272 (50\%) of patients were accompanied. A total of 238 (43\%) patients presented after a fall, which was the commonest reason for attendance. Four hundred and one $(73 \%)$ patients were seen by senior house officers, $91(16 \%)$ by an A\&E consultant or registrar and $40(7 \%)$ by emergency nurse practitioners.

\section{FOLLOW UP BY CLN}

The CLN contacted 258 (46\%) patients on the first attempt. The remainder required up to four telephone calls to make contact. The CLN was initially unable to contact 80 (14\%) patients. Within this group contact was made with one or more of the following: GPs surgery (52), health visitor (5), district nursing service (2) or other agencies (36). In 11 cases no contact could be made, most of these patients were from outside of the local area.

\section{PRE-EXISTING HOME CIRCUMSTANCES}

The majority of patients $322(58 \%)$ were supported at home by friends or family, 143 (26\%) received support from other agencies including voluntary services and a smaller group 61 $(11 \%$ ) had home help, input from a community nurse $50(9 \%)$, social services $48(9 \%)$ or received meals on wheels eight $(1.5 \%)$. The type of accommodation was recorded in 121 $(22 \%)$ of the original $\mathrm{A} \& \mathrm{E}$ notes and was wrongly recorded in 15 of these.

ADVICE AND REFERRAL BY CLN

Before discharge, advice had been given to 514 (93\%) patients in the A\&E department. This 


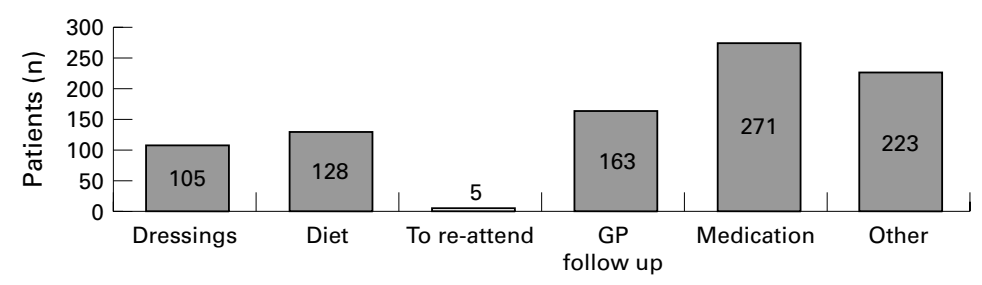

Figure 1 Advice given by the community liaison nurse.

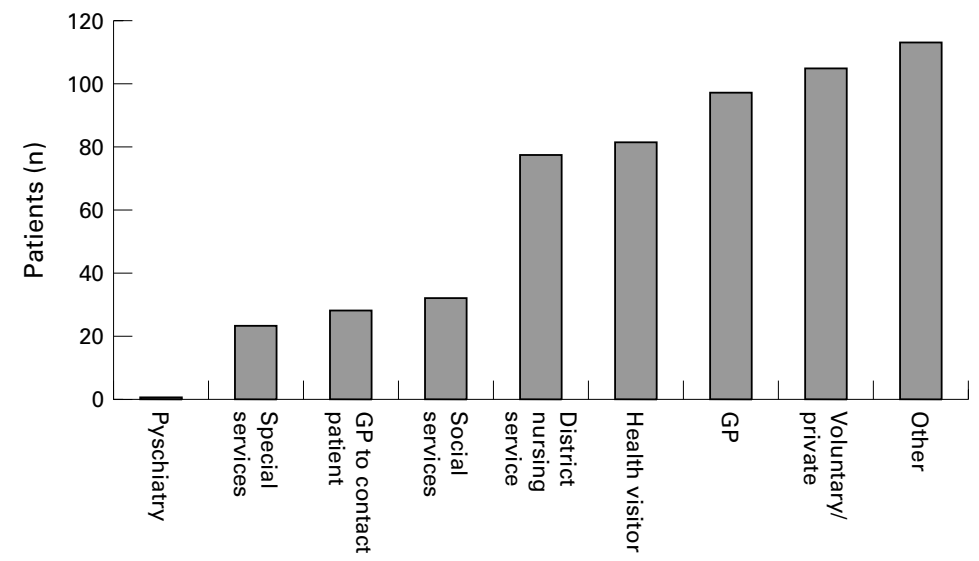

Figure 2 Referrals made by the community liaison nurse.

was written in $169(33 \%)$ cases and verbal in $331(64 \%)$ cases. Advice was given by the CLN on a broad range of issues including medication, follow up, occupational therapy, diet and dressings (fig 1). Health education given by the CLN was understood by 381 (69\%) patients and $457(83 \%)$ understood the follow up advice given. Patients who failed to understand the advice given were targeted for early follow up by a health visitor. Existing home support was felt to be insufficient in 44 $(8 \%)$ cases and in need of immediate intervention in a further $45(8 \%)$ cases. Eighty four $(15 \%)$ patients were taking incorrect or inadequate medication. A total of 559 referrals were made by the CLN after telephone assessment (fig 2). Some patients had more than one referral. One hundred and twenty seven (23\%) patients were visited at home by their general practitioner, health visitor, district nurse, community pharmacist or other agencies (fig 3). A wide range of health care services were accessed, ranging from specialist services such as community diabetes, stoma and asthma nurses to local or national support and self help groups such as Age Concern, Help the Aged, The Alzheimer's Disease Society, The Carers

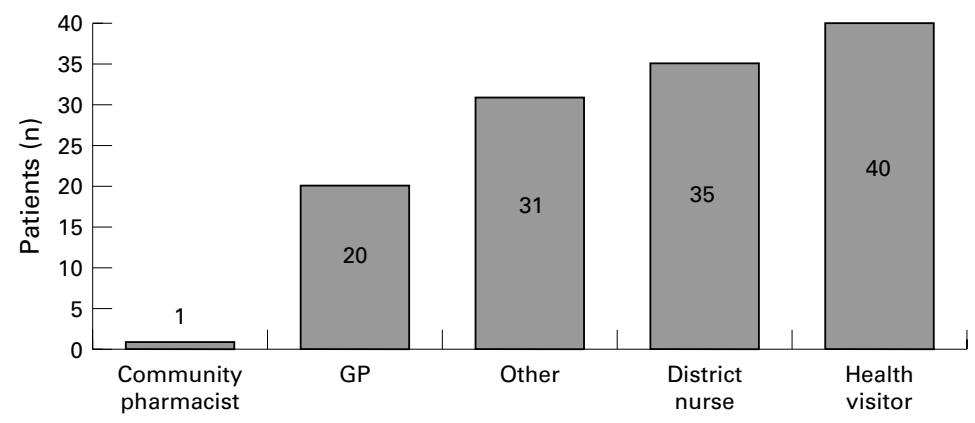

Figure 3 Agents who visited patients at home.
Association and Arthritis Care. An emergency response was requested by the CLN in six cases.

\section{Discussion}

East Sussex has one of the largest elderly populations per capita in the UK with over 165000 patients aged over 75, many of whom have complex medical and psychosocial problems. ${ }^{14}$ One in 20 over the age of 70 and one in five over the age of 80 suffer from dementia. Furthermore, elderly people with diminished cognitive function may be socially isolated because of communication and mobility problems. These problems may easily be overlooked when patients are seen by busy, inexperienced doctors or nurses who may fail to appreciate the significance of seemingly minor complaints. For instance, abnormal symptoms such as incontinence or mild confusion may be regarded as "normal". Achieving a rapid, accurate, functional and social assessment of elderly patients within an A\&E department is a recognised problem. ${ }^{6}{ }^{15}$ History taking may indeed be difficult, however collaborative histories from a relative, neighbour or GP are often equally important and frequently omitted.

CRITICAL INCIDENT IDENTIFICATION*

Within our department this tool has enabled us to identify a number of problems with service provision for this vulnerable group of patients; junior medical and nursing staff had difficulties pre-empting patients needs on discharge as well as identifying the most appropriate community service provider. Patients were often sent home with inadequate home support. Poor documentation was a recurrent theme. The type of accommodation was omitted in the majority of the original A\&E notes. The presence or absence of stairs was also a frequently omitted detail. Without such basic information it is arguably impossible to form an accurate or useful social assessment. Similarly, information regarding known existing contacts with social services or the community nursing service was also frequently omitted. The type and quality of information given to our elderly patients was variable and often of poor quality. Treatments and medications were often prescribed for elderly patients without ensuring patients' comprehension. Poor pain control was a frequently encountered problem. Changes in medication initiated by the $A \& E$ department were often not maintained after the A\&E prescription had finished. Patients were occasionally issued with repeat prescriptions by their GP who may have been unaware of medication changes made within the $A \& E$ department.

The need for immediate help is unrelated to whether the patient is already receiving assistance. It is vital not to accept social situations at face value whether a person lives alone or with the family. ${ }^{15}$ Discharge coordination from the A\&E department may be overlooked in a high

* Critical incident: any event that caused or had the potential of causing an adverse clinical outcome. 
pressure, high workload, environment. Services already available for dependent elderly patients are often underused, leaving family, carers, friends or neighbours to provide the majority of essential help. This study showed that social support needs often go unrecognised or tend to be greatly underestimated during initial assessment, and these may in turn lead to critical incidents. The study also identified that staff are often unaware of what avenues of support already exist within the local community or how to access them, especially when patients attend outside of normal working hours. In response to critical incidents that were identified we have improved our educational programmes for both medical and nursing staff. Furthermore, vulnerable elderly patients are not sent home after $9 \mathrm{pm}$ and are admitted overnight to our short stay ward for multidisciplinary assessment the following morning. We have encouraged broader use of computer generated patient advice letters.

\section{NURSE LED TELEPHONE FOLLOW UP}

A telephone follow up service requires a nurse with communication skills as well as in depth knowledge of local community medical and social services. ${ }^{5}$ Unexpected emotional responses may be triggered, elderly patients may also have hearing difficulties and a clear sensitive telephone manner is needed to give necessary information without distressing the patient. We found that patients were often not prepared for follow up. Cold calling should be avoided if possible by warning patients that they should expect a follow up telephone call from the hospital.

\section{ADVANTAGES OF NEXT DAY TELEPHONE FOLLOW} UP

A telephone callback system is a feasible and effective method to improve follow up care of elder patients released from the $A \& E$ department. ${ }^{16}$ Minor problems that are neglected may easily develop leading to reattendance, admission to acute general medical wards or long term care facilities, complaints and critical incidents. Next day telephone follow up may help to diminish these problems by facilitating the identification and assessment of those in need, determining whether a home visit is necessary and enabling other early targeted interventions. It also allows nurses to offer support for patients themselves to cope with minor self limiting conditions, to recognise any worsening or previously undiagnosed symptoms, to check patient understanding of advice given and to inform patients about when and where to seek further follow up. This results in better informed patients and helps to prevent problems developing into crises. A telephone follow up system is a practical tool for monitoring and detecting high risk patients, furthermore it provides $A \& E$ staff with data that can be used in quality improvement audits, in developing post-release protocols and improving patient satisfaction with the care received in the $\mathrm{A} \& \mathrm{E}$ department. ${ }^{16}$ It is also a low cost service requiring only a single nurse with telephone access for five half days per week.

\section{Conclusion}

The concept of discharge planning for elderly patients leaving the A\&E department needs re-evaluation. Innovative approaches to discharge planning that incorporate social and personal as well as medical and nursing needs should be developed. ${ }^{2}$ We recommend next day telephone follow up of patients over 75 as a low cost, high quality service, which allows the identification of high risk patients and focused interventions. By targeting at risk groups at an early stage it is possible to identify and perhaps prevent critical incidents and so has the potential for decreasing inappropriate return visits by a group of patients with a relatively high dependency. This tool identifies deficiencies within the $A \& E$ department. It helps to bridge the gap that exists between $A \& E$ and primary health care services. It improves patient health education and highlights the important issues involved in discharge coordination that allows us to re-focus our teaching for medical and nursing staff. It is a dynamic ongoing system and continued monitoring of trends will enable us to refocus our service delivery for this vulnerable group of patients.

Contributors

HP discussed core ideas, analysed and interpreted the data and wrote the paper. JR initiated the study, designed the protocol and participated in data analysis and writing the paper. MC helped design the study, collected the data and participated in writing the paper. JR is guarantor for the paper.

Funding: none.

Conflicts of interest: none.

1 Family Policies Studies Centre. Factsheet 1: Putting Families on the map. London: FPSC, 1994.

2 Sanders AB. Care of the elderly in emergency departments: conclusions and recommendations. Ann Emerg Med 1992;21:830-4.

3 Singal BM, Hedges JR, Rousseau EW, et al. Geriatric patient emergency visits. Part 1: Comparison of geriatric and younger patients. Ann Emerg Med 1992;21:802-7.

4 Howard R. Reasons for older people attending A\&E. Nursing Times 1995; 13:40-3.

ing Times 1995;13:40-3.
Allen D. Telephone follow up for older people discharged Allen D. Telephone follow up for older people
from A\&E. Nursing Standard 1997;46:34-7.

from A\&E. Nursing Standard 1997;46:34-7.
6 Runciman P, Currie CT, Nicol M, et al. Discharge of elderly people from an accident and emergency department: evaluation of health visitor follow-up. F Adv Nurs 1996;24: 711-18.

7 Rowland K, Maitra AK, Richardson A, et al. The discharge of elderly patients from an accident and emergency department: functional changes and risk of re-admission. Age Ageing 1990;19:415-18.

8 Currie CT, Lawson PM, Robertson CE, et al. Elderly patients discharged from an accident and emergency department: their dependency and support. Arch Emerg Med 1984;1:205-13.

9 National Audit Commission for local authorities \& the NHS. By accident or design: Improving A $\&$ E services in England $\mathcal{E}$ Wales. London: HMSO, 1996.

10 The Health of the Nation: A strategy for health in England. London: HMSO, 1992.

11 Hendriksen C, Lund E, Stromgard E. Consequences of assessment and intervention among elderly people: a three year randomised controlled trial. BMF 1984;289:1522-4.

12 Denman SJ, Ettinger WH, Zarkin BA, et al. Short-term outcomes of elderly patients discharged from an emergency department. I Am Geriatr Soc 1989;37:937-43.

13 Tremellan J. Assessing the elderly in the community. Health Visitor 1990;63:49-51.

14 Cryer C, Davidson L, Styles C. Injury epidemiology in the southeast: identifying priorities for action. Bexhill: South East Institute of Public Health, 1993.

15 Dove AF, Dave SH, Gerrard E. The accident department and Age Concern. Health Trends 1986;18:86-8.

16 Jones J, Young M, Lafleur R, et al. Effectiveness of an organised follow-up system for elder patients released from the Emergency Department. Acad Emerg Med 1997;4:114752 . 